ISSN 0258-7122

Bangladesh J. Agril. Res. 37(1): 137-148, March 2012

\title{
RESPONSE OF MUSTARD (Brassica) VARIETIES TO BORON APPLICATION
}

\author{
M. A. HOSSAIN ${ }^{1}$, M. JAHIRUDDIN ${ }^{2}$ AND F. KHATUN $^{3}$
}

\begin{abstract}
An experiment was conducted at the Regional Agricultural Research Station (RARS), Jessore (AEZ11, High Ganges River Floodplain) during 2003-2006 to evaluate the response of different varieties of mustard to boron application. Boron application was made at 0 and $1 \mathrm{~kg} / \mathrm{ha}$. The mustard varieties responded to B application. The response of the three Brassica species followed the order: $B$. napus $>B$. campestris $>B$. juncea. The varieties chosen from $B$. campestris were BARI Sarisha 6, BARI Sarisha 9, and BARI Sarisha 12. The B. napus varieties were BARI Sarisha 7, BARI Sarisha 8, and BARI Sarisha 13. Varieties BARI Sarisha 10 and BARI Sarisha 11 were from the $B$. juncea group. The seed yield was positively and significantly correlated with the yield contributing characters viz. pods/plant, seeds/pod, and 1000-seed weight, but not with plant height and pod length. This result showed that boron had positive influence on reproductive development, not on vegetative. The result suggests that BARI Sarisha 10 and BARI Sarisha 11 were the most B in-responsive (B efficient) varieties. So the farmers can grow these varieties in the moderately $B$ deficient soils with a minimum dose $(0.5 \mathrm{~kg} / \mathrm{ha})$ of B application.
\end{abstract}

Keywords: Mustard, boron, B in-responsive.

\section{Introduction}

Among the oilseed crops, mustard is the major oilseed crop, which covers about $60 \%$ of the oilseed production in Bangladesh (BBS, 2009). It is an important source of cooking oil in Bangladesh and it meets one third of the edible oil requirement of the country (Ahmed et al., 1988). The average yield of the crop stands at $990 \mathrm{~kg} / \mathrm{ha}$ (BBS, 2009), which is very low compared to the yield of many mustard growing countries of the world. There are several reasons that can explain this yield variation, which cover abiotic and biotic factors. Among the biotic and abiotic factors, unavailability of high yielding varieties (Akber et al., 1994) and nutrient deficiency (Varma et al., 2002) are responsible for lower productivity of mustard. The newly released high yielding potential varieties of mustard could not compensate the yield gap possibly due to B deficiency in soil. Mustard, a Brassica crop, is very responsive to B application (Mengel and Kirkby, 1987). Reproductive growth, especially flowering, fruit and seed set is

\footnotetext{
${ }^{1}$ Principal Scientific Officer, Pulses Research Centre, Bangladesh Agricultural Research Institute (BARI), Gazipur, ${ }^{2}$ Professor, Dept. of Soil Science, Bangladesh Agricultural University (BAU), Mymensingh and ${ }^{3}$ Senior Scientific Officer, Regional Agricultural Research Station (BARI), Jessore, Bangladesh.
} 
more sensitive to B deficiency than vegetative growth (Dear and Lipsett, 1987). Boron requirement for root growth in B - in efficient rapeseed cultivars was higher than that in B efficient cultivars (Hu et al., 1994; Xioug et al., 1995).

Availability of B to plants is affected by a variety of soil factors including soil solution, $\mathrm{pH}$, texture, moisture, temperature, oxide content, carbonate content, organic matter content, and clay mineralogy (Goldberg et al., 2000). Boron is generally less available in high $\mathrm{pH}$ soil. Increasing $\mathrm{pH}$ favours its retention by soils or soil constituents (Mezuman and Karen, 1981; Bloesch et al., 1987; Goldberg, 1997). Jessore is an extensively mustard growing area and the soil of this area is calcareous in nature. Mustard varieties may differ in its sensitivity to B deficiency. Keeping the above points in view, the present study was undertaken to evaluate the response of mustard varieties to B application.

\section{Materials and Method}

Eight varieties of mustard viz. BARI Sarisha 6, BARI Sarisha 7, BARI Sarisha 8, BARI Sarisha 9, BARI Sarisha 10, BARI Sarisha 11, BARI Sarisha 12, and BARI sarisha 13 were tested for their response to B application (0 and $1 \mathrm{~kg}$ $\mathrm{B} / \mathrm{ha}$ ). Of the eight varieties, three were from Brassica campestries, three from Brassica napus and two from Brassica juncea. Crop response to added B was evaluated in terms of yield and yield contributing characters. The experiment was conducted in the calcareous soil of Regional Agricultural Research Station (RARS) farm, Jessore, Bangladesh for 3 years from 2003-2004 to 2005-2006. The land belongs to High Ganges River Floodplain agroecological zone (11) and Gopalpur soil series (Soil taxonomy: Aquic Eutro-chrepts). The soil had high $\mathrm{pH}$ value (8.1) and with low B content of $0.18 \mathrm{mg} / \mathrm{kg}$. The organic matter content of the soil was $1.65 \%$, Olsen-P $10.1 \mathrm{mg} / \mathrm{kg}$, exchangeable K 0.28 c mol $/ \mathrm{kg}$, Ca 19 c $\mathrm{mol} / \mathrm{kg}, \mathrm{Mg} 1.75 \mathrm{c} \mathrm{mol} / \mathrm{kg}, \mathrm{CaCl}_{2}-\mathrm{S} 4.53 \mathrm{mg} / \mathrm{kg}$ and DTPA-Zn $0.89 \mathrm{mg} / \mathrm{kg}$. Soil $\mathrm{P}^{\mathrm{H}}$ was determined by glass electrode $\mathrm{pH}$ meter (1:2.5 soil-water ratio) and organic matter by wet oxidation method (Nelson and Sommers, 1982). The K, $\mathrm{Ca}$, and $\mathrm{Mg}$ contents of soil were determined by $1 \mathrm{M} \mathrm{NH}_{4} \mathrm{OAc}\left(\mathrm{P}^{\mathrm{H}} 7.0\right)$ extraction method. The experiment was laid out in a randomized complete block design with three replications, each plot size being $4 \times 3 \mathrm{~m}$. Boron was applied at 0 and 1 $\mathrm{kg} / \mathrm{ha}$. This layout was kept undisturbed for the second and third years of the study. Boron was supplied as $\mathrm{H}_{3} \mathrm{BO}_{3}(17 \% \mathrm{~B})$. Every year, the mustard crop received $124 \mathrm{~kg} \mathrm{~N}, 30 \mathrm{~kg} \mathrm{P}, 28 \mathrm{~kg} \mathrm{~K}, 35 \mathrm{~kg} \mathrm{~S}$ and $2 \mathrm{~kg} Z \mathrm{Zn}$ per hectare (BARC, 2005). The sources of nutrients were urea, triple super phosphate (TSP), muriate of potash (MoP), gypsum, and zinc sulphate for $\mathrm{N}, \mathrm{P}, \mathrm{K}, \mathrm{S}$, and $\mathrm{Z}$, respectively. Intercultural operations viz., weeding, irrigation, and insecticide spray were done as and when required. Every year, the mustard varieties were sown during first week of November and harvested in the second week of February. At maturity, data on the yield contributing characters were recorded from ten randomly 
selected plants from each plot. The yield data were expressed as $\mathrm{kg} / \mathrm{ha}$ on $12 \%$ moisture basis. The data were statistically analyzed following the principle of $F$ statistics and the mean values were separated by Duncan's Multiple Range Test (DMRT) (Gomez and Gomez, 1984)

\section{Results and Discussion}

\section{Yield components}

Different mustard varieties significantly influenced on the number of pods/plant due to B application except BARI Sarisha 10. The number of pods/plant of $B$. campestris variety varied from 104.0 to $183.1, B$. napus 133.3 to 139.9 , and $B$. juncea 251.2 to 267.7 in the B treated plots against 80.56 to $143.0,91.89$ to 102.4, and 242.9 to 249.2 in the control plots of the respective three varieties. The highest number of pods/plant increased $45.1 \%$ in the BARI Sarisha 8 and the lowest 3.42\% in BARI Sarisha 10 due to B application (Table 1). Except first year trial, BARI Sarisha 11 produced the highest number of pods/plant followed by BARI Sarisha 10. BARI Sarisha 7, BARI Sarisha 8, and BARI Sarisha 13 produced statistically similar number of pods/plant in the control plots. However, the effect of $B$ on these three varieties was significantly higher than all other varieties. This result proved that $B$. napus is most responsive to $\mathrm{B}$ followed by $B$. campestries. Brassica juncea is merely responsive to B. On the basis of pods/plant, different varieties can be ranked as BARI Sarisha 11> BARI Sarisha 10> BARI Sarisha 12> BARI Sarisha 9> BARI Sarisha 7> BARI Sarisha 13> BARI Sarisha 8> BARI Sarisha 6. Shen et al. (1993) reported that B application markedly increased the number of pods/plant in with rape cv. Ningyou No. 8 and Ningyou No.7.

The number of seeds/pod also varied significantly among the varieties due to $\mathrm{B}$ application (Table 2). The average number of seeds/pod ranged from 12.00 to 20.67 and 13.22 to 27.44 in the B untreated and treated plots, respectively. The maximum average number of seeds/pod (27.44) was recorded in B treated BARI Sarisha 8, which was $32.8 \%$ higher than that where B was not added to the soil. The application of B failed to increase significantly the number of seeds/pod in BARI Sarisha 10. On the basis of number of seeds/pod, the Brassica groups can be ranked as $B$. napus $>B$. campestris $>B$. juncea. This finding corroborate with the findings of Shen et al., 1993; Islam and Sarker (1993), and Hu et al., 1994.

In the case of B. campestris group, 1000-seed weight varied from 3.12 to $3.76 \mathrm{~g}, \mathrm{~B}$. napus group 3.76 to $3.89 \mathrm{~g}$ and $B$. juncea group 2.10 to $3.92 \mathrm{~g}$ due to $\mathrm{B}$ application, whereas it was 2.59 to $3.16 \mathrm{~g}$ in $B$. campestris group, 3.08 to $3.21 \mathrm{~g}$ in $B$. napus group and 2.03 to $3.63 \mathrm{~g}$ in $B$. juncea group in the B untreated plots. BARI Sarisha 8 of the B. napus variety, produced $24.0 \%$ higher 1000 -seed 
weight over B control plot; whereas, BARI Sarisha 10 increased only 3.45\% due to B application (Table 3). BARI Sarisha 11 of Brassica juncea variety produced the highest weight of 1000 seeds (3.92 g) in the B treated plot, on the other hand, B. juncea variety BARI Sarisha 10 produced lowest 1000 -seeds weight (2.10 g) in the B control plot.

Table 1. Number of pods/plant of different varieties of mustard due to boron application.

\begin{tabular}{|c|c|c|c|c|c|c|c|}
\hline \multirow[b]{2}{*}{ Group } & \multirow[b]{2}{*}{ Variety } & \multirow{2}{*}{$\begin{array}{c}\text { Boron } \\
\text { level } \\
\text { (kg/ha) }\end{array}$} & \multicolumn{4}{|c|}{ Pods/plant (no.) } & \multirow{2}{*}{$\begin{array}{c}\% \\
\text { increase } \\
\text { over } \\
\text { control }\end{array}$} \\
\hline & & & 2003-04 & 2004-05 & 2005-06 & Average & \\
\hline \multirow{6}{*}{$\begin{array}{c}\text { Brassica } \\
\text { campestris }\end{array}$} & \multirow[t]{2}{*}{$\mathrm{V}_{1}$} & $\mathrm{~B}_{0}$ & $89.00 \mathrm{~g}$ & $85.00 \mathrm{j}$ & $67.67 \mathrm{~h}$ & 80.56 & - \\
\hline & & $\mathrm{B}_{1}$ & $110.3 \mathrm{f}$ & 112.0gh & $92.00 \mathrm{~g}$ & 104.8 & 30.1 \\
\hline & \multirow[t]{2}{*}{$\mathrm{V}_{2}$} & $\mathrm{~B}_{0}$ & 132.3e & $119.3 g$ & 99.33g & 117.0 & - \\
\hline & & $\mathrm{B}_{1}$ & $162.0 \mathrm{~d}$ & $146.0 \mathrm{f}$ & 130.3cde & 146.1 & 24.9 \\
\hline & \multirow[t]{2}{*}{$\mathrm{V}_{3}$} & $\mathrm{~B}_{0}$ & 147.0de & $167.0 \mathrm{e}$ & 115.0ef & 143.0 & - \\
\hline & & $\mathrm{B}_{1}$ & 191.0c & 211.0d & 147.3c & 183.1 & 28.0 \\
\hline \multirow{6}{*}{$\begin{array}{c}\text { Brassica } \\
\text { napus }\end{array}$} & \multirow[t]{2}{*}{$\mathrm{V}_{4}$} & $\mathrm{~B}_{0}$ & 109.3f & 100.0hi & $98.00 \mathrm{~g}$ & 102.4 & - \\
\hline & & $\mathrm{B}_{1}$ & $145.0 \mathrm{e}$ & $138.7 f$ & $136.0 \mathrm{~cd}$ & 139.9 & 36.6 \\
\hline & \multirow[t]{2}{*}{$\mathrm{V}_{5}$} & $\mathrm{~B}_{0}$ & 97.33fg & $92.00 \mathrm{ij}$ & 86.33g & 91.89 & - \\
\hline & & $\mathrm{B}_{1}$ & $135.0 \mathrm{e}$ & $135.7 f$ & 129.3cde & 133.3 & 45.1 \\
\hline & \multirow[t]{2}{*}{$\mathrm{V}_{6}$} & $\mathrm{~B}_{0}$ & 103.0fg & 98.33hij & $93.00 \mathrm{~g}$ & 98.1 & - \\
\hline & & $\mathrm{B}_{1}$ & 135.3e & 137.0f & 135.3cd & 135.9 & 38.5 \\
\hline \multirow{5}{*}{$\begin{array}{c}\text { Brassica } \\
\text { juncea }\end{array}$} & \multirow[t]{2}{*}{$\mathrm{V}_{7}$} & $\mathrm{~B}_{0}$ & $265.7 \mathrm{a}$ & 238.7c & $224.3 b$ & 242.9 & - \\
\hline & & $\mathrm{B}_{1}$ & $273.7 \mathrm{a}$ & $248.0 c$ & 232.0b & 251.2 & 3.42 \\
\hline & \multirow[t]{2}{*}{$\mathrm{V}_{8}$} & $\mathrm{~B}_{0}$ & $247.0 \mathrm{~b}$ & $272.0 \mathrm{~b}$ & 228.7b & 249.2 & - \\
\hline & & $\mathrm{B}_{1}$ & 261.7a & 293.3a & $248.0 \mathrm{a}$ & 267.7 & 7.42 \\
\hline & & CV (\%) & 5.56 & 4.89 & 6.78 & - & - \\
\hline
\end{tabular}

Values within the same column with a common letter do not differ significantly $(\mathrm{p}=0.05)$

$\mathrm{V}_{1}$ = BARI Sarisha 6, $\mathrm{V}_{2}$ = BARI Sarisha 9, $\mathrm{V}_{3}$ = BARI Sarisha 12, $\mathrm{V}_{4}$ = BARI Sarisha 7, $\mathrm{V}_{5}=$ BARI Sarisha 8, $V_{6}=$ BARI Sarisha 13, $V_{7}=$ BARI Sarisha 10, $V_{8=}$ BARI Sarisha 11

Pod length varied significantly among the varieties due to B application (Table 4). The maximum average pod length $(8.31 \mathrm{~cm})$ was recorded in BARI Sarisha 13 , which was statistically identical to BARI Sarisha $8(8.17 \mathrm{~cm})$. In B control plots, highest pod length also observed in BARI Sarisha $13(7.79 \mathrm{~cm})$ being followed by BARI sarisha $8(7.44 \mathrm{~cm})$. The minimum pod length was recorded in B untreated BARI Sarisha $10(4.11 \mathrm{~cm})$ and BARI Sarisha $11(4.11 \mathrm{~cm})$. 
The impact of B application on plant height of mustard was positive to some extent but not significant (Table 5). Boron application increased mean plant height from $2.48 \%$ in BARI Sarisha 10 to $8.17 \%$ in BARI Sarisha 13 . The maximum average plant height $(160.9 \mathrm{~cm})$ was recorded in B added BARI Sarisha 11 variety, but it was only $100.3 \mathrm{~cm}$ in B untreated BARI Sarisha 7. Islam and Sarker (1993) reported that boron application significantly increased the number of pods/plant, seeds/pod and seed yield of mustard. Hu et al. (1994) reported that B application at the rate of $0.1 \mathrm{mg} / \mathrm{kg}$ soil was optimum at which plant height, branch number/plant, siliqua number/plant, seed number/siliqua, seed yield/plant, and oil yield/plant increased from $149.2 \mathrm{~cm}, 14.1,302.3,12.6,14.51 \mathrm{~g}$ and $5.63 \mathrm{~g}$ in the control to $158.0 \mathrm{~cm}, 23.8,400.2,16.3,20.93 \mathrm{~g}$ and $9.61 \mathrm{~g}$, respectively.

Table 2. Number of seeds/pod of different varieties of mustard due to boron application.

\begin{tabular}{|c|c|c|c|c|c|c|c|}
\hline \multirow{2}{*}{ Group } & \multirow{2}{*}{ Variety } & \multirow{2}{*}{$\begin{array}{c}\text { Boron } \\
\text { level } \\
\text { (kg/ha) }\end{array}$} & \multicolumn{4}{|c|}{ Seeds/pod (no.) } & \multirow{2}{*}{$\begin{array}{c}\% \text { increase } \\
\text { over } \\
\text { control }\end{array}$} \\
\hline & & & 2003-04 & 2004-05 & 2005-06 & Average & \\
\hline \multirow{6}{*}{$\begin{array}{c}\text { Brassica } \\
\text { campestris }\end{array}$} & \multirow[t]{2}{*}{$\mathrm{V}_{1}$} & $\mathrm{~B}_{0}$ & $21.00 \mathrm{e}$ & $20.67 c$ & 19.00c & 20.22 & - \\
\hline & & $\mathrm{B}_{1}$ & $26.00 \mathrm{~b}$ & 25.67ab & $24.33 b$ & 25.33 & 25.3 \\
\hline & \multirow[t]{2}{*}{$\mathrm{V}_{2}$} & $\mathrm{~B}_{0}$ & 14.33i & 14.00gh & $13.00 \mathrm{e}$ & 13.78 & - \\
\hline & & $\mathrm{B}_{1}$ & $17.67 \mathrm{gh}$ & $17.00 \mathrm{f}$ & $15.67 d$ & 16.78 & 2.18 \\
\hline & \multirow[t]{2}{*}{$\mathrm{V}_{3}$} & $\mathrm{~B}_{0}$ & $16.33 \mathrm{~h}$ & 15.33g & $15.67 d$ & 15.78 & - \\
\hline & & $\mathrm{B}_{1}$ & $19.00 \mathrm{~g}$ & 17.33ef & $18.33 c$ & 18.22 & 15.5 \\
\hline \multirow{6}{*}{$\begin{array}{c}\text { Brassica } \\
\text { napus }\end{array}$} & \multirow[t]{2}{*}{$\mathrm{V}_{4}$} & $\mathrm{~B}_{0}$ & $19.33 f$ & $18.67 d$ & $19.67 \mathrm{c}$ & 19.22 & - \\
\hline & & $\mathrm{B}_{1}$ & $25.00 \mathrm{bc}$ & $24.33 b$ & $24.33 b$ & 24.55 & 27.7 \\
\hline & \multirow[t]{2}{*}{$\mathrm{V}_{5}$} & $\mathrm{~B}_{0}$ & 23.33d & $20.00 \mathrm{~cd}$ & $18.67 \mathrm{e}$ & 20.67 & - \\
\hline & & $\mathrm{B}_{1}$ & 29.67a & 26.33a & 26.33a & 27.44 & 32.8 \\
\hline & \multirow[t]{2}{*}{$\mathrm{V}_{6}$} & $\mathrm{~B}_{0}$ & $19.67 \mathrm{df}$ & 19.33cd & $19.00 \mathrm{c}$ & 19.33 & - \\
\hline & & $\mathrm{B}_{1}$ & 24.33cd & 25.67ab & $24.67 \mathrm{~b}$ & 24.89 & 28.8 \\
\hline \multirow{5}{*}{$\begin{array}{c}\text { Brassica } \\
\text { juncea }\end{array}$} & \multirow[t]{2}{*}{$\mathrm{V}_{7}$} & $\mathrm{~B}_{0}$ & $14.33 \mathrm{i}$ & $12.67 \mathrm{~h}$ & $12.67 \mathrm{e}$ & 13.22 & - \\
\hline & & $\mathrm{B}_{1}$ & $14.67 \mathrm{i}$ & 13.33h & 13.33e & 13.78 & 4.25 \\
\hline & \multirow[t]{3}{*}{$\mathrm{V}_{8}$} & $\mathrm{~B}_{0}$ & $12.33 \mathrm{j}$ & $12.67 \mathrm{~h}$ & $11.00 \mathrm{f}$ & 12.0 & - \\
\hline & & $\mathrm{B}_{1}$ & $12.67 \mathrm{j}$ & 14.00gh & $13.00 \mathrm{e}$ & 13.22 & 10.2 \\
\hline & & CV (\%) & 4.29 & 4.40 & 4.76 & - & - \\
\hline
\end{tabular}

Values within the same column with a common letter do not differ significantly $(\mathrm{p}=0.05)$

$\mathrm{V}_{1}=$ BARI Sarisha 6, $\mathrm{V}_{2}$ = BARI Sarisha 9, $\mathrm{V}_{3}$ = BARI Sarisha 12, $\mathrm{V}_{4}=$ BARI Sarisha 7,

$\mathrm{V}_{5}=$ BARI Sarisha 8, $\mathrm{V}_{6}=$ BARI Sarisha 13, $\mathrm{V}_{7}=$ BARI Sarisha 10, $\mathrm{V}_{8=}$ BARI Sarisha 11

\section{Seed yield}

Except BARI Sarisha 10, all the mustard varieties was significantly favoured by boron application with the ranking of $B$. napus $>B$. campestris $>B$. juncea (Table 6 ). The $B$. campestris varieties yielded $1671-1795 \mathrm{~kg} / \mathrm{ha}$ (mean of 3 years), $B$ 
Table 3. Thousand seed weight of different varieties of mustard due to boron application.

\begin{tabular}{|c|c|c|c|c|c|c|c|}
\hline \multirow{2}{*}{ Group } & \multirow{2}{*}{ Variety } & \multirow{2}{*}{$\begin{array}{c}\text { Boron } \\
\text { level } \\
\text { (kg/ha) }\end{array}$} & \multicolumn{4}{|c|}{ 1000-seed weight (g) } & \multirow{2}{*}{$\begin{array}{c}\% \text { increase } \\
\text { over } \\
\text { control }\end{array}$} \\
\hline & & & 2003-04 & 2004-05 & 2005-06 & Average & \\
\hline \multirow{6}{*}{$\begin{array}{c}\text { Brassica } \\
\text { campestris }\end{array}$} & \multirow[t]{2}{*}{$\mathrm{V}_{1}$} & $\mathrm{~B}_{0}$ & 3.34de & $3.17 f$ & $2.97 c$ & 3.16 & - \\
\hline & & $\mathrm{B}_{1}$ & $3.84 b$ & 3.93ab & $3.50 \mathrm{~b}$ & 3.76 & 19.0 \\
\hline & \multirow[t]{2}{*}{$\mathrm{V}_{2}$} & $\mathrm{~B}_{0}$ & 2.99g & $2.29 \mathrm{~h}$ & $2.48 d$ & 2.59 & - \\
\hline & & $\mathrm{B}_{1}$ & $3.45 \mathrm{~cd}$ & $2.81 \mathrm{~g}$ & $3.11 c$ & 3.12 & 20.5 \\
\hline & \multirow[t]{2}{*}{$\mathrm{V}_{3}$} & $\mathrm{~B}_{0}$ & $3.06 \mathrm{fg}$ & $3.07 f$ & $2.89 c$ & 3.01 & - \\
\hline & & $\mathrm{B}_{1}$ & $3.53 c$ & $3.47 \mathrm{a}$ & $3.41 \mathrm{~b}$ & 3.47 & 15.3 \\
\hline \multirow{6}{*}{$\begin{array}{c}\text { Brassica } \\
\text { napus }\end{array}$} & \multirow[t]{2}{*}{$\mathrm{V}_{4}$} & $\mathrm{~B}_{0}$ & $3.27 \mathrm{e}$ & 3.19ef & $2.96 c$ & 3.14 & - \\
\hline & & $\mathrm{B}_{1}$ & $3.94 b$ & $3.87 b$ & $3.46 \mathrm{~b}$ & 3.76 & 19.7 \\
\hline & \multirow[t]{2}{*}{$\mathrm{V}_{5}$} & $\mathrm{~B}_{0}$ & $3.08 \mathrm{fg}$ & 3.24def & $2.91 \mathrm{c}$ & 3.08 & - \\
\hline & & $\mathrm{B}_{1}$ & $3.92 b$ & 3.93ab & $3.62 b$ & 3.82 & 24.0 \\
\hline & \multirow[t]{2}{*}{$\mathrm{V}_{6}$} & $\mathrm{~B}_{0}$ & $3.20 \mathrm{ef}$ & $3.43 c d$ & $3.00 c$ & 3.21 & - \\
\hline & & $\mathrm{B}_{1}$ & 4.01ab & $4.12 \mathrm{a}$ & $3.55 b$ & 3.89 & 21.3 \\
\hline \multirow{4}{*}{$\begin{array}{c}\text { Brassica } \\
\text { juncea }\end{array}$} & \multirow[t]{2}{*}{$\mathrm{V}_{7}$} & $\mathrm{~B}_{0}$ & $2.07 \mathrm{~h}$ & $2.09 \mathrm{i}$ & $1.94 \mathrm{e}$ & 2.03 & - \\
\hline & & $\mathrm{B}_{1}$ & $2.13 \mathrm{~h}$ & 2.12hi & $2.06 \mathrm{e}$ & 2.10 & 3.45 \\
\hline & \multirow[t]{2}{*}{$\mathrm{V}_{8}$} & $\mathrm{~B}_{0}$ & 3.99ab & 3.39cde & $3.52 b$ & 3.63 & - \\
\hline & & $\mathrm{B}_{1}$ & $4.11 \mathrm{a}$ & $3.75 b$ & $3.90 \mathrm{a}$ & 3.92 & 7.99 \\
\hline CV (\%) & & & 2.58 & 3.56 & 4.34 & - & - \\
\hline
\end{tabular}

Values within the same column with a common letter do not differ significantly $(\mathrm{p}=0.05)$ $\mathrm{V}_{1}=$ BARI Sarisha 6, $\mathrm{V}_{2}=$ BARI Sarisha 9, $\mathrm{V}_{3}$ = BARI Sarisha 12, $\mathrm{V}_{4}=$ BARI Sarisha 7, $\mathrm{V}_{5}=$ BARI Sarisha 8, $\mathrm{V}_{6}=$ BARI Sarisha 13, $\mathrm{V}_{7}=$ BARI Sarisha 10, $\mathrm{V}_{8=}$ BARI Sarisha 11

napus $1917-2020 \mathrm{~kg} / \mathrm{ha}$ and $B$. juncea gave $1763-2151 \mathrm{~kg} /$ ha yield due to B addition to soil. The corresponding yields from the control plots were 1359-1509, 1397-1544 and 1734-1971 kg/ha, respectively (Table 6). Considering percent yield increase over control (mean of 3 years) Brassica napus varieties showed a 25.62 to $30.83 \%$ yield increase, Brassica campestris had a 19.02 to $22.96 \%$ yield increase and Brassica juncea varieties exhibited a 1.67 to $9.17 \%$ yield increase over B control. BARI Sarisha 8 exhibited the highest B response and BARI Sarisha 10 did the lowest indicating that the former was highly sensitive and the latter was fairly tolerant to B deficiency. Nevertheless, the Brassica juncea varieties gave higher seed yield compared to the other groups particularly when boron was not applied. 
Table 4. Pod length of different varieties of mustard due to boron application.

\begin{tabular}{|c|c|c|c|c|c|c|c|}
\hline \multirow{2}{*}{ Group } & \multirow{2}{*}{ Variety } & \multirow{2}{*}{$\begin{array}{c}\text { Boron } \\
\text { level } \\
\text { (kg/ha) }\end{array}$} & \multicolumn{4}{|c|}{ Pod length $(\mathrm{cm})$} & \multirow{2}{*}{$\begin{array}{c}\% \text { increase } \\
\text { over } \\
\text { control }\end{array}$} \\
\hline & & & 2003-04 & 2004-05 & 2005-06 & Average & \\
\hline \multirow{6}{*}{$\begin{array}{c}\text { Brassica } \\
\text { campestris }\end{array}$} & \multirow[t]{2}{*}{$\mathrm{V}_{1}$} & $\mathrm{~B}_{0}$ & 5.95e & $5.97 d$ & $5.22 \mathrm{~g}$ & 5.71 & - \\
\hline & & $\mathrm{B}_{1}$ & $6.20 \mathrm{~d}$ & $6.23 \mathrm{~d}$ & $5.50 \mathrm{f}$ & 5.98 & 4.27 \\
\hline & \multirow[t]{2}{*}{$\mathrm{V}_{2}$} & $\mathrm{~B}_{0}$ & $4.57 \mathrm{~h}$ & $4.70 f$ & $4.23 \mathrm{j}$ & 4.50 & - \\
\hline & & $\mathrm{B}_{1}$ & $4.82 \mathrm{~g}$ & $4.94 \mathrm{ef}$ & $4.59 \mathrm{i}$ & 4.78 & 6.22 \\
\hline & \multirow[t]{2}{*}{$\mathrm{V}_{3}$} & $\mathrm{~B}_{0}$ & $5.65 f$ & $4.94 \mathrm{ef}$ & $4.88 \mathrm{~h}$ & 5.16 & - \\
\hline & & $\mathrm{B}_{1}$ & 5.78ef & 5.17e & $5.15 g$ & 5.37 & 4.07 \\
\hline \multirow{6}{*}{$\begin{array}{c}\text { Brassica } \\
\text { napus }\end{array}$} & \multirow[t]{2}{*}{$\mathrm{V}_{4}$} & $\mathrm{~B}_{0}$ & $6.42 \mathrm{~d}$ & $6.18 d$ & $5.95 e$ & 6.18 & - \\
\hline & & $\mathrm{B}_{1}$ & $6.73 c$ & $6.73 c$ & 6.34d & 6.60 & 6.80 \\
\hline & \multirow[t]{2}{*}{$\mathrm{V}_{5}$} & $\mathrm{~B}_{0}$ & $7.67 \mathrm{~b}$ & $7.57 b$ & $7.09 \mathrm{c}$ & 7.44 & - \\
\hline & & $\mathrm{B}_{1}$ & $8.28 \mathrm{a}$ & $8.42 \mathrm{a}$ & $7.80 \mathrm{~b}$ & 8.17 & 9.81 \\
\hline & \multirow[t]{2}{*}{$\mathrm{V}_{6}$} & $\mathrm{~B}_{0}$ & $7.82 \mathrm{~b}$ & $7.88 \mathrm{~b}$ & $7.68 b$ & 7.79 & - \\
\hline & & $\mathrm{B}_{1}$ & 8.32a & $8.42 \mathrm{a}$ & $8.20 \mathrm{a}$ & 8.31 & 6.68 \\
\hline \multirow{5}{*}{$\begin{array}{c}\text { Brassica } \\
\text { juncea }\end{array}$} & \multirow[t]{2}{*}{$\mathrm{V}_{7}$} & $\mathrm{~B}_{0}$ & $4.26 \mathrm{i}$ & $4.04 \mathrm{~g}$ & $4.02 \mathrm{j}$ & 4.11 & - \\
\hline & & $\mathrm{B}_{1}$ & 4.34hi & $4.16 \mathrm{~g}$ & $4.08 \mathrm{j}$ & 4.19 & 1.95 \\
\hline & \multirow[t]{3}{*}{$\mathrm{V}_{8}$} & $\mathrm{~B}_{0}$ & $4.12 \mathrm{i}$ & $4.09 \mathrm{~g}$ & $4.11 \mathrm{j}$ & 4.11 & - \\
\hline & & $\mathrm{B}_{1}$ & $4.24 \mathrm{i}$ & $4.28 \mathrm{~g}$ & $4.29 \mathrm{j}$ & 4.27 & 3.89 \\
\hline & & CV (\%) & 2.47 & 2.84 & 2.80 & - & - \\
\hline
\end{tabular}

Values within the same column with a common letter do not differ significantly $(\mathrm{p}=0.05)$

$\mathrm{V}_{1}=$ BARI Sarisha 6, $\mathrm{V}_{2}=$ BARI Sarisha 9, $\mathrm{V}_{3}=$ BARI Sarisha 12, $\mathrm{V}_{4}=$ BARI Sarisha 7 , $\mathrm{V}_{5}=$ BARI Sarisha 8, $\mathrm{V}_{6}=$ BARI Sarisha 13, $\mathrm{V}_{7}=$ BARI Sarisha 10, $\mathrm{V}_{8=}$ BARI Sarisha 11

Response of mustard yield to boron application has been reported by many researchers in the past (Banuelos et al., 1993; Shen et al., 1993; Bora and Hazarika, 1997; Lu et al., 2000; Xue et al., 1998). Rashid et al. (1994) reported the maximum seed yield increase for Brassica napus (43\%) is at $1 \mathrm{~kg} / \mathrm{ha} \mathrm{B}$ application and for Brassica juncea (36\%) at $1.5 \mathrm{~kg} \mathrm{~B} / \mathrm{ha}$ rate. Xue et al. (1998) reported that the significant differences were found among the cultivars in leaf boron concentration. Contrary to current opinion, high quality oilseed rape cultivars were not all sensitive to low B supply nor were all conventional cultivars B efficient. There are significant differences between Brassica napus cultivars in their response to B deficiency (Hu et al., 1991; James et al., 2000a,b). 
Table 5. Plant height of different varieties of mustard due to boron application.

\begin{tabular}{|c|c|c|c|c|c|c|c|}
\hline \multirow[b]{2}{*}{ Group } & \multirow[b]{2}{*}{ Variety } & \multirow{2}{*}{$\begin{array}{l}\text { Boron } \\
\text { level } \\
\text { (kg/ha) }\end{array}$} & \multicolumn{4}{|c|}{ Plant height (cm) } & \multirow{2}{*}{$\begin{array}{c}\% \text { increase } \\
\text { over } \\
\text { control }\end{array}$} \\
\hline & & & $\begin{array}{c}2003- \\
04 \#\end{array}$ & $\begin{array}{c}2004- \\
05 \#\end{array}$ & $\begin{array}{c}2005- \\
06 \#\end{array}$ & Average & \\
\hline \multirow{6}{*}{$\begin{array}{c}\text { Brassica } \\
\text { campestris }\end{array}$} & \multirow[t]{2}{*}{$\mathrm{V}_{1}$} & $\mathrm{~B}_{0}$ & 152.4 & 125.8 & 119.7 & 132.6 & - \\
\hline & & $\mathrm{B}_{1}$ & 154.5 & 130.5 & 126.6 & 137.2 & 3.47 \\
\hline & \multirow[t]{2}{*}{$\mathrm{V}_{2}$} & $\mathrm{~B}_{0}$ & 111.8 & 89.4 & 101.7 & 101.0 & - \\
\hline & & $\mathrm{B}_{1}$ & 119.4 & 96.1 & 109.8 & 108.4 & 7.33 \\
\hline & \multirow[t]{2}{*}{$\mathrm{V}_{3}$} & $\mathrm{~B}_{0}$ & 118.8 & 103.6 & 107.9 & 110.1 & - \\
\hline & & $\mathrm{B}_{1}$ & 123.5 & 110.5 & 114.5 & 116.2 & 5.54 \\
\hline \multirow{6}{*}{$\begin{array}{c}\text { Brassica } \\
\text { napus }\end{array}$} & \multirow[t]{2}{*}{$\mathrm{V}_{4}$} & $\mathrm{~B}_{0}$ & 110.2 & 99.2 & 91.6 & 100.3 & - \\
\hline & & $\mathrm{B}_{1}$ & 115.2 & 101.0 & 98.9 & 105.0 & 4.69 \\
\hline & \multirow[t]{2}{*}{$\mathrm{V}_{5}$} & $\mathrm{~B}_{0}$ & 124.4 & 98.3 & 99.8 & 107.5 & - \\
\hline & & $\mathrm{B}_{1}$ & 133.6 & 106.1 & 107.2 & 115.6 & 7.53 \\
\hline & \multirow[t]{2}{*}{$\mathrm{V}_{6}$} & $\mathrm{~B}_{0}$ & 116.6 & 109.6 & 104.5 & 110.2 & - \\
\hline & & $\mathrm{B}_{1}$ & 124.2 & 121.2 & 112.3 & 119.2 & 8.17 \\
\hline \multirow{5}{*}{$\begin{array}{l}\text { Brassica } \\
\text { juncea }\end{array}$} & \multirow[t]{2}{*}{$\mathrm{V}_{7}$} & $\mathrm{~B}_{0}$ & 156.1 & 141.0 & 126.3 & 141.1 & - \\
\hline & & $\mathrm{B}_{1}$ & 160.0 & 144.6 & 129.3 & 144.6 & 2.48 \\
\hline & \multirow[t]{3}{*}{$\mathrm{V}_{8}$} & $\mathrm{~B}_{0}$ & 167.7 & 155.3 & 138.9 & 154.0 & - \\
\hline & & $\mathrm{B}_{1}$ & 171.4 & 165.1 & 146.2 & 160.9 & 4.48 \\
\hline & & CV (\%) & 5.95 & 6.36 & 6.53 & - & - \\
\hline
\end{tabular}

\# Non= Non significant

$\mathrm{V}_{1}$ = BARI Sarisha 6, $\mathrm{V}_{2}=$ BARI Sarisha 9, $\mathrm{V}_{3}$ = BARI Sarisha 12, $\mathrm{V}_{4}=$ BARI Sarisha 7, $\mathrm{V}_{5}=$ BARI Sarisha 8, $\mathrm{V}_{6}=$ BARI Sarisha 13, $\mathrm{V}_{7}=$ BARI Sarisha 10, $\mathrm{V}_{8=}$ BARI Sarisha 11

Table 6. Seed yield of different varieties of mustard due to boron application.

\begin{tabular}{|c|c|c|c|c|c|c|c|}
\hline \multirow[b]{2}{*}{ Group } & \multirow[b]{2}{*}{ Variety } & \multirow{2}{*}{$\begin{array}{c}\text { Boron } \\
\text { level } \\
\text { (kg/ha) }\end{array}$} & \multicolumn{4}{|c|}{ Seed yield (kg/ha) } & \multirow{2}{*}{$\begin{array}{c}\% \\
\text { increase } \\
\text { over } \\
\text { control }\end{array}$} \\
\hline & & & 2002-03 & 2003-04 & 2004-05 & Average & \\
\hline \multirow{6}{*}{$\begin{array}{c}\text { Brassica } \\
\text { campestris }\end{array}$} & $\mathrm{V}_{1}$ & $\mathrm{~B}_{0}$ & $1686 c-f$ & $1671 d-g$ & $1170 \mathrm{~h}$ & 1509 & - \\
\hline & & $\mathrm{B}_{1}$ & 1948b & 1973ab & 1467efg & 1795 & 19.02 \\
\hline & $\mathrm{V}_{2}$ & $\mathrm{~B}_{0}$ & 1487fg & 1311h & 1280gh & 1359 & - \\
\hline & & $\mathrm{B}_{1}$ & 1774bcd & 1600 efg & 1640de & 1671 & 22.96 \\
\hline & $\mathrm{V}_{3}$ & $\mathrm{~B}_{0}$ & 1469g & 1495g & 1308gh & 1424 & - \\
\hline & & $\mathrm{B}_{1}$ & 1756b-e & 1783b-e & 1547def & 1695 & 19.03 \\
\hline
\end{tabular}


Table 6 Cont'd.

\begin{tabular}{|cccccccc|}
\hline $\begin{array}{c}\text { Brassica } \\
\text { napus }\end{array}$ & $\mathrm{V}_{4}$ & $\mathrm{~B}_{0}$ & $1450 \mathrm{~g}$ & $1565 \mathrm{fg}$ & $1177 \mathrm{~h}$ & 1397 & - \\
& & $\mathrm{B}_{1}$ & $1877 \mathrm{bc}$ & $1939 \mathrm{abc}$ & $1577 \mathrm{def}$ & 1998 & 28.70 \\
& $\mathrm{~V}_{5}$ & $\mathrm{~B}_{0}$ & $1629 \mathrm{~d}-\mathrm{g}$ & $1615 \mathrm{efg}$ & $1389 \mathrm{fg}$ & 1544 & - \\
& & $\mathrm{B}_{1}$ & $2150 \mathrm{a}$ & $2062 \mathrm{a}$ & $1847 \mathrm{bc}$ & 2020 & 30.83 \\
& $\mathrm{~V}_{6}$ & $\mathrm{~B}_{0}$ & $1549 \mathrm{efg}$ & $1711 \mathrm{def}$ & $1318 \mathrm{gh}$ & 1526 & - \\
& & $\mathrm{B}_{1}$ & $1944 \mathrm{~b}$ & $2109 \mathrm{a}$ & $1698 \mathrm{~cd}$ & 1917 & 25.62 \\
\hline \multirow{2}{*}{$\begin{array}{c}\text { Brassica } \\
\text { juncea }\end{array}$} & $\mathrm{V}_{7}$ & $\mathrm{~B}_{0}$ & $1827 \mathrm{bcd}$ & $1762 \mathrm{c}-\mathrm{f}$ & $1612 \mathrm{de}$ & 1734 & - \\
& & $\mathrm{B}_{1}$ & $1896 \mathrm{bcd}$ & $1742 \mathrm{c}-\mathrm{f}$ & $1652 \mathrm{de}$ & 1763 & 1.67 \\
& & $\mathrm{~B}_{0}$ & $2152 \mathrm{a}$ & $1854 \mathrm{bcd}$ & $1906 \mathrm{~b}$ & 1971 & - \\
& $\mathrm{B}_{1}$ & $2257 \mathrm{a}$ & $2080 \mathrm{a}$ & $2115 \mathrm{a}$ & 2151 & 9.17 \\
\hline
\end{tabular}

Values within the same column with a common letter do not differ significantly $(\mathrm{p}=0.05)$ $\mathrm{V}_{1}$ = BARI Sarisha 6, $\mathrm{V}_{2}$ = BARI Sarisha 9, $\mathrm{V}_{3}$ = BARIsarisha 12, $\mathrm{V}_{4}$ = BARI Sarisha 7, $\mathrm{V}_{5}$ $=$ BARI Sarisha 8, $\mathrm{V}_{6}=$ BARI Sarisha 13, $\mathrm{V}_{7}=$ BARI Sarisha 10, $\mathrm{V}_{8=}$ BARI Sarisha 11

Table 7. Stover yield of different varieties of mustard due to boron application.

\begin{tabular}{|c|c|c|c|c|c|c|c|}
\hline \multirow[b]{2}{*}{ Group } & \multirow[b]{2}{*}{ Variety } & \multirow{2}{*}{$\begin{array}{c}\text { Boron } \\
\text { level } \\
\text { (kg/ha) }\end{array}$} & \multicolumn{4}{|c|}{ Stover yield (kg/ha) } & \multirow{2}{*}{$\begin{array}{c}\% \text { increase } \\
\text { over } \\
\text { control }\end{array}$} \\
\hline & & & $\begin{array}{c}2002-03 \\
\#\end{array}$ & $\begin{array}{c}\text { 2003-04 } \\
\#\end{array}$ & $\begin{array}{c}2004-05 \\
\#\end{array}$ & Average & \\
\hline \multirow{6}{*}{$\begin{array}{c}\text { Brassica } \\
\text { campestris }\end{array}$} & \multirow[t]{2}{*}{$\mathrm{V}_{1}$} & $\mathrm{~B}_{0}$ & 2993 & 2656 & 2456 & 2702 & - \\
\hline & & $\mathrm{B}_{1}$ & 3189 & 2704 & 2595 & 2829 & 4.07 \\
\hline & \multirow[t]{2}{*}{$\mathrm{V}_{2}$} & $\mathrm{~B}_{0}$ & 2372 & 2171 & 2129 & 2224 & - \\
\hline & & $\mathrm{B}_{1}$ & 2542 & 2301 & 2216 & 2353 & 5.80 \\
\hline & \multirow[t]{2}{*}{$\mathrm{V}_{3}$} & $\mathrm{~B}_{0}$ & 2366 & 2382 & 2179 & 2309 & - \\
\hline & & $\mathrm{B}_{1}$ & 2528 & 2592 & 2360 & 2493 & 8.25 \\
\hline \multirow{6}{*}{$\begin{array}{c}\text { Brassica } \\
\text { napus }\end{array}$} & \multirow[t]{2}{*}{$\mathrm{V}_{4}$} & $\mathrm{~B}_{0}$ & 2614 & 3234 & 2703 & 2850 & - \\
\hline & & $\mathrm{B}_{1}$ & 2736 & 3246 & 2988 & 2990 & 4.91 \\
\hline & \multirow[t]{2}{*}{$\mathrm{V}_{5}$} & $\mathrm{~B}_{0}$ & 3055 & 3610 & 2933 & 3199 & - \\
\hline & & $\mathrm{B}_{1}$ & 3307 & 4077 & 3226 & 3537 & 10.57 \\
\hline & \multirow[t]{2}{*}{$\mathrm{V}_{6}$} & $\mathrm{~B}_{0}$ & 2600 & 3382 & 2807 & 2930 & - \\
\hline & & $\mathrm{B}_{1}$ & 2973 & 3579 & 2936 & 3163 & 7.95 \\
\hline \multirow{5}{*}{$\begin{array}{c}\text { Brassica } \\
\text { juncea }\end{array}$} & $\mathrm{V}_{7}$ & $\mathrm{~B}_{0}$ & 3058 & 3191 & 2985 & 3080 & - \\
\hline & \multirow{4}{*}{$\mathrm{V}_{8}$} & $\mathrm{~B}_{1}$ & 3269 & 3380 & 3069 & 3239 & 5.16 \\
\hline & & $\mathrm{B}_{0}$ & 3564 & 3849 & 3172 & 3528 & - \\
\hline & & $\mathrm{B}_{1}$ & 3622 & 4119 & 3300 & 3680 & 4.30 \\
\hline & & CV (\%) & 5.70 & 6.50 & 6.14 & - & - \\
\hline
\end{tabular}

\# = Non-significant

$\mathrm{V}_{1}=$ BARI Sarisha 6, $\mathrm{V}_{2}=$ BARI Sarisha 9, $\mathrm{V}_{3}$ = BARI Sarisha 12, $\mathrm{V}_{4}=$ BARI Sarisha 7, $\mathrm{V}_{5}=$ BARI Sarisha 8, $\mathrm{V}_{6}=$ BARI Sarisha 13, $\mathrm{V}_{7}=$ BARI Sarisha 10, $\mathrm{V}_{8=}$ BARI Sarisha 11 


\section{Stover yield}

Unlike seed yield, the stover yield remained unaffected by B treatments (Table 7). Apparently, BARI Sarisha 8 (Brassica napus) had the maximum response to B application, on the other hand, BARI Sarisha 11 (Brassica juncea) showed the minimum response. The mean yields of $B$. campestris varieties were 2224-2702 $\mathrm{kg} / \mathrm{ha}, B$. napus varieties were $2850-3199 \mathrm{~kg} / \mathrm{ha}$, and yields of $B$. juncea varieties were $3080-3528 \mathrm{~kg} / \mathrm{ha}$ for the B control plots. The yields for the B added treatments were $2353-2829 \mathrm{~kg} / \mathrm{ha}, 2990-3537 \mathrm{~kg} / \mathrm{ha}$ and 3239-3680 kg/ha, respectively. Dear and Lipsett (1987) opined that reproductive growth is more sensitive to B deficiency than vegetative growth. Saha et al. (2003) reported that the use of B did not influence the stover yield of mustard.

\section{Conclusion}

Based on the information mentioned above, it may be concluded that BARI Sarisha 10 and BARI Sarisha 11 (B. juncea) were non-responsive to B application but are well suited to the Gopalpur series soil of Jessore to give appreciably higher yield of mustard without application of B fertilizer. BARI Sarisha 7, BARI Sarisha 8 and BARI Sarisha 13 (B. napus) were responsive to B application and can be grown in Gopalpur series soil by applying B @ 1.0 kg/ha, though the yield is low.

\section{References}

Ahmed, M. S., M. Elias and J. Ahmed. 1988. Results of farm level socio-economic study on mustard production. Bangladesh J. Agril. Res. 13(1): 20-23.

Akber, A., M. Mondal, P. Podder and H. Ahmed. 1994. Sarisha Phasholer Chash- A booklet in Bengali. Oilseed Research Centre, BARI, Joydebpur, Gazipur.

Banuelos, G.S., G.E. Cardon, C.J. Phene, L. Wu, S. Akohoue and S.Zambrzuski. 1993. Soil boron and selenium removal by three plant species. Plant and Soil. 148(2): 253-263.

BARC. 2005. Fertilizer Recommendation Guide-2005. BARC Pub. No. 45. Bangladesh Agricultural Research Council, Dhaka.

BBS. 2009. Year Book of Agricultural Statistics of Bangladesh. Bangladesh Bureau of Statistics, Ministry of Planning, Govt. of the People's Republic of Bangladesh, Dhaka.

Bloesch, P.M., L.C. Bell and J.D. Hughes. 1987. Adsorption and desorption of boron by goethite. Aust. J. Soil Res. 25: 377-390.

Bora, P.C. and U. Hazarika. 1997. Effect of lime and boron on rainfed toria (Brassica campestris subsp. Oleifera var. Toria). Indian J. Agron. 42 (2): 361-364.

Dear, B.S. and J. Lipsett. 1987. The effect of boron supply on the growth and seed production of subterranean clover (Trifolium subterraneum L.). Aust. J. Agric. Res. 38: 537-546. 
Goldberg, S., M.L. Scott and D.L. Suarez. 2000. Predicting boron adsorption by soils using soil chemical parameters in the constant capacitance model. Soil Sci. Soc. Am. J. 64: 1356-1363.

Gomez, K.A. and A.A. Gomez. 1984. Statistical Procedures for Agricultural Research. Int. Rice Res. Inst., John Wiley \& Sons, NY.

Goldberg S. 1997. Reaction of boron with soils. Plant and Soil. 193: 35-48.

Hu, B., Z. Jiang and Y. Zeng. 1991. Slurry sample introduction with fluorinating electrothermal vaporization for the direct ICP-AES determination of boron in plant leaves (Brassica napus L.). Oil Crops (China) 16(3): 43-46.

Hu, Y.S., Y.H. Ma, Y.L. Sun and G. Guo. 1994. Effect of B application on the agronomic traits, yield and oil content of a double-low rape (Brassica napus L.) cultivar. Oil Crops (China). 16(3): 43-46.

Islam, M.B. and M.A.Z. Sarker. 1993. Effects of different levels of boron on the yield of mustard. Research Report 1992-1993. Bangladesh Agricultural Research Institute, On-farm Research Division, Agricultural Research Station, Rangpur. pp. 1-4

James, C. R. S., S.H. Grewal, R.W. Bell and R.D.Graham. 2000a. Boron efficiency in oilseed rape: I. Genotypic variation demonstrated in field and pot grown Brassica napus L., and Brassica juncea L. Plant and Soil 225: 243-251.

James, C. R. S., S.H. Grewal, R.W. Bell and R.D. Graham. 2000b. Boron efficiency in oilseed rape: II. Development of a rapid-based screening technique. Plant and Soil 225: 253-261.

Lu, X., Y. Lu, D. Mao, Y.F. Lu. and D.M. Mao. 2000. The levels of available boron in red earth in Central Zhejiang Provice and boron nutrition of rape. Institute of Soils and Fertilizers, Beijing. 5(1): 30-31.

Mengel, K. and E.A. Kirkby. 1987. Principles of Plant Nutrition. International Potash Institute, Switzerland.

Mezuman, U. and R. Karen. 1981. Boron adsorption by soils using a phenomenological adsorption equation. Soil Sci. Soc. Am. J. 45: 722-726.

Nelson, D.W. and L.E. Sommers. 1982. Total Carbon, organic carbon and organic matter, In: Methods of Soil Analysis, Part 2. $2^{\text {nd }}$ Ed. Page, A.L. Miller, R.H. and Keeney, D.R. Amer. Soc. Agron. Inc., Madi., Wis., USA. pp. 539-580.

Rashid, A., E.Rafique and N. Bughio. 1994. Diagnosing boron deficiency in rapeseed and mustard by plant analysis and soil testing. Commun. Soil Sci. Plant Anal. 25(17/18): 2283-2897.

Saha, P.K., M.A. Saleque, S.K. Zaman and N.I. Bhuiyan. 2003. Response of mustard to S, Zn and B in calcareous soil. Bangladesh J. Agril. Res. 28(4): 633-636.

Shen, K., Z.G., Shen, H.Q. Xu and Q.Y. Huang. 1993. A study of boron nutrition and seed setting in rape (Brassica napus). Acta Agronomica Sinica. 19 (6): 539-545. 
Varma, S.C., R.K. Srivastava, S. Rajesh, S.K. Singh, P.K. Bisen and R. Singh. 2002. Effect of varying levels of sulphur and potassium on yield and oil content of Indian mustard (Brassica Juncea). Research on Crops. 3(3): 650-652.

Xiong, S., L. Wu, Y. Wang and L. Lan. 1995. Absorption and distribution of boron of different rape cultivars. J. Huazhong Agril. Univ. (Supplement) 21: 85-91.

Xue, J., L. Minshu, W. Richard, R.D. Bell, X.Y. Graham and Y. Yuai. 1998. Deferential response of oilseed rape (Brassica napus L.) cultivars to low boron supply. Plant and Soil. 204(2): 155-163. 